\title{
Epigenetics Communications: where are we?
}

\author{
Lucia Altucci ${ }^{1}$ and Marianne G. Rots ${ }^{2^{*}}$
}

Welcome to the first volume of Epigenetics Communications, which as the companion journal to Clinical Epigenetics provides a platform for exchanging insights on epigenetic mechanisms that impact functioning of a wide spectrum of systems and organisms, before and beyond clinical applications. Interviews with our expert associate editors will be posted on the website soon, and some statements reflecting their perspectives on the journal and towards this rapidly growing epigenetic field can be found below.

The control of gene expression via a convoluted regulatory machinery operating "above the genome" intrinsically implies the complexity of epigenetics and epigenome functions and dysfunctions. Indeed, exploiting the continuously improving genomics technology portfolio, epigenome-wide association studies have yielded extensive databases, with nucleotide resolution and single cell precision, at increasingly affordable costs. These insights, covering various organisms, have indicated differences but also similarities across species. Bringing together different disciplines, as stimulated by Epigenetics Communications, is expected to result in increased understanding of functional epigenomics. As we can deduce thus far, some of the regulatory epinetworks are reversible but at the same time can leave a "memory"; this concept underlines the potential of epigenetics to control (patho)physiological mechanism(s) underlying functions in all kingdoms of life.

With the scientific and societal boom raised in recent years by epigenetic studies, there is the yet unmet need to clarify fundamental basic epi-mechanisms and to interfere with their regulation and function(s). The

\footnotetext{
*Correspondence: m.g.rots@umca.nl

${ }^{2}$ University of Groningen, University Medical Center Groningen, Hanzeplein 1, 9713GZ, Groningen, the Netherlands

Full list of author information is available at the end of the article
}

CRISPR revolution has sped up such developments, as nuclease-inactivated Cas9 proteins can be fused to epigenetic writers/erasers to interfere with epigenetic modifications at any given chromatin context. Apart from essential scientific insights, such epigenetic editing studies have resulted in agricultural and preclinical therapeutic successes.

While epigenetic modifications have now been "mined" and functionally studied singularly, the epigenetic systems and networks-and their (de)regulationsare still far from being understood: their intrinsic dynamic nature and the potentially "quick" transitions still represent technological challenges and consequently scientific bottlenecks. Furthermore, the so-called epiplayers (regulating eu- to heterochromatin transitions and vice versa) are switching between chromatin-bound and unbound states. Moreover, chromatin modifications show not only rapid states changes but have intrinsic deposition or transition order with functional consequences. These epidynamics transitions (and their potential order) within chromatin, the spatial deconvolution, and the impact on genome functioning are yet to be fully understood, as well as their impact on the adaptation of these states in any given organism.

Thus, the interpretation of the dynamic epigenome and the spatiotemporal organization in different biological systems might be a crucial layer of knowledge in physiology and pathology in different species at a basic as well as preclinical/biotechnological level. Indeed, epigenetics can be exploited in all kingdoms of life to adjust phenotypes, ranging from improving model organisms to economical applications (agricultural, environmental, personal care, etc.).

Unveiling the networks of the epi-modulatory events and their precise order by novel (computational) methods for identification and analyses for example at the single cell holds the key for understanding the 
regulation of gene expression, causal (and not casual) order of cellular events leading to chromatin organization and at the same time stability and maintenance of cellular function. The study of the network epigenetics may advance our mechanistic understanding of the epi-dynamics within the 3D structure of the cell and of tissues. These issues have dedicated sections in Epigenetics Communications.

In addition, chromatin epi-regulators may take advantage of the abundance of metabolic co-factors, supporting the concept that availability of those cofactors might determine to some extent prioritization of events. Integration of basic epigenome information with dynamics and metabolic status of the cells might bridge basic to preclinical information in the fight against diseases.

Given the explosion of epigenetic-based studies, it is of crucial importance to distinguish reproducible vs irreproducible approaches as well as technically sound studies that "fail" to prove a scientific hypothesis. Epigenetics Communications will act as a forum to showcase and discuss alternative conclusions/interpretations of wellestablished epigenetic phenomena based on (re-)analyses of published or new data. As such, we welcome "systematic studies," be it positive (expected) or negative (nonexpected) with respect to its outcome.

Publication of these so called negative data (for details, see our website: https://epicom.biomedcentral.com/ ? gclid = Cj0KCQiA 4b 2 MBhD 2 ARIs A IrcB-Rdl3 e9ibIOFfjw9jRr_xgKJJ4TG49TzWirhfmTY38miZJXZ4 aAz7gaAkuSEALw_wcB)) will benefit the science and scientists by ensuring sharing of epi-data. It will foster increased technical and scientific knowledge communication within the field. With its basic and multidisciplinary approach, Epigenetics Communications not only highlights sound albeit "negative" data publishing as a relevant base for understanding epigenetic regulation but also intends to actively boost the re-interpretations of epigenome data and their outcomes.

The integration of the epi-based data and systems with spatial and temporal information (and their intercommunications in a network context) will represent the next challenge where data knowledge, technology challenges, and the scientific integration will be of paramount relevance. We aim to promote such expected developments by welcoming submissions, board members, and guest editors. So far, we recruited a broad panel of experts to cover the different sections: a sneak preview on their commitments is given here; the extended interviews will be published on the website.

Q: What are you particularly excited about with the launch of Epigenetics Communications?
A: "The journal will be filling an open gap in the community and providing a space for important studies that contribute to the further understanding of epigenetics which do not have specific clinical implications yet. We can use them to build something larger and something ultimately that will have clinical impacts." (Kimberly Glass)

A: "It is an opportunity to help define a niche in this vast research field. It will create a platform where the community can work out, clarify and conceptualise very specific epigenetic phenomena that would probably otherwise not get as much appreciation than in other journals, which is very exciting." (Frank Johannes)

A: "I am very excited about publishing our articles in a specific journal on Epigenetics, which are usually to be put in different journals. We could easily get an overview of the Epigenetics research in the field through EPIC." (Frank Dekker)

Q: How do you feel about Epigenetics Communications providing of a platform for "null hypothesisconfirming" results?

A: "That's a new thing. Negative results are quite common in research and often hard to publish. This information is also very valuable for others in the community as they can save time by not repeating the same. It could be a good complement, given they are testing interesting hypothesis and have high standard results." (Karl Ekwall)

A: "I think it is interesting and important because sometimes you have data that do not confirm the hypothesis, but it is thorough and wellperformed. Publishing this type of data is a new attempt, but it needs critical reviewing. This is a challenge if the hypothesis goes into a different direction, but it could actually be a really interesting new finding to get further follow up." (Pernette J. Verschure)

A: "It is very nice that we have a platform to publish all the relevant findings that are negative. There are so many people doing different experiments and they are often fear of showing what they expect. It is a very important part of the scientific process to also publish these wrong expectations and negative data. This helps people in the field in the same way as positive data." (Torsten Plösch) 
Q: Do you think peer review is critical to research and why? Do you foresee a serious competition by platforms like bioRxiv?

A: "I think bioRxiv is interesting because it has the flexibility to already put out your data and to have comments by other researchers. However, peer reviewing is still critical because there are so many data out there and how researchers can keep track on everything if you do not know whether it is trustable data and all of these." (Pernette J. Verschure)

A: "I think the current peer review system is not ideal yet. It is very interesting to see we have platforms where we can publish manuscripts and everybody can add comments. This is very open, just like social media, so it is very hard to regulate. I believe that the honest and good comments from colleagues are the best way to judge and help authors to publish good manuscript." (Torsten Plösch)

A: "I think peer review is really crucial and you could put anything on bioRxiv without any quality control. It is essential to have peer review for EPIC, as the system works very well in molecular biology and should not be abandoned. The colleagues who are experts in the field can actually judge the quality before it is published." (Karl Ekwall)

Q: How do you see this field changing in the coming 5 to 10 years?

A: "With this preponderance of data, we will see a recognition of the complexity of biological systems and the need to integrate across different types of data. To fully embrace the complexity, we should look at how everything works together and how this leads to something larger like the network and the emergent properties of a system." (Kimberley Glass)

A: "With the advancement of mass spectrometry and sequencing technologies, novel epimodifications/epimarks will continue to be revealed. It will be an exciting quest for the ongoing discovery of novel epimodifications and hopefully in the near future we will conquer these uncharted epi(c) territories and be able to monitor the global epi-omic signature of individuals. In this regard, the ability to decipher and manipulate the epi-omes might present new avenues e.g. in drug design for procuring better treatment of various human diseases." (Andy Lau)
A: "The field is getting broader as epigenetics have impacts on many things. I think data science will play a more important role with the large initiative to map the epigenome. New drug targets will be discovered, together with new pathways and new links to new diseases. These all in all will bring innovations in diagnosis and therapy out." (Frank Dekker)

A: "The field would still be very much dominated by technological advances with major advances in the single cell epigenomic sequencing assays. They will provide unprecedented insights into an individual's functional variation across different cell types and development. The drop in sequencing cost will also allow us to scale up epigenomic sequencing projects to population level surveys." (Frank Johannes)

\section{Acknowledgements}

We are very grateful to Vanessa Tse (University of Bath, UK) for conducting the interviews.

Authors' contributions

The authors have written the text and approved the final manuscript.

\section{Competing interests}

Both authors serve as Editors-in-Chief of Clinical Epigenetics, as well as of Epigenetics Communications.

\section{Author details}

'Department of Precision Medicine, University of Campania, Luigi Vanvitelli, Via L. De Crecchio 7, 80138 Naples, Italy. ${ }^{2}$ University of Groningen, University Medical Center Groningen, Hanzeplein 1, 9713GZ, Groningen, the Netherlands.

Published online: 20 December 2021

\section{Publisher's Note}

Springer Nature remains neutral with regard to jurisdictional claims in published maps and institutional affiliations.

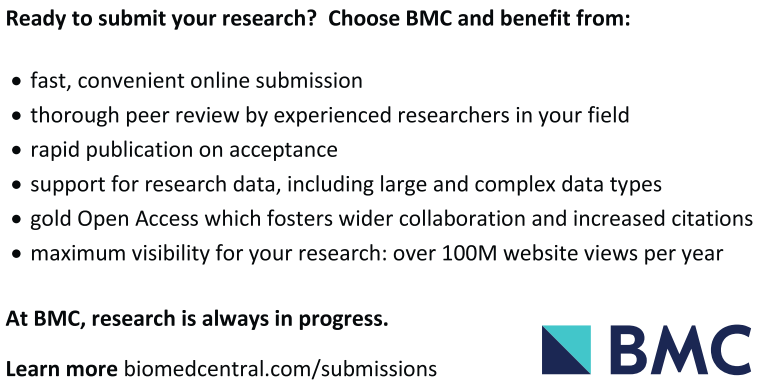

